\title{
GENERIC AND SUBTRIBAL RELATIONSHIPS IN NEOTROPICAL CYMBIDIEAE (ORCHIDACEAE) BASED ON MATK/YCF1 PLASTID DATA
}

\author{
W. Mark Whitten ${ }^{1,2}$, Kurt M. Neubig ${ }^{1} \&$ N. H. Williams ${ }^{1}$ \\ ${ }^{1}$ Florida Museum of Natural History, University of Florida \\ Gainesville, FL 32611-7800 USA \\ ${ }^{2}$ Corresponding author: whitten@flmnh.ufl.edu
}

\begin{abstract}
AвSTRACT. Relationships among all subtribes of Neotropical Cymbidieae (Orchidaceae) were estimated using combined matK/ycfl plastid sequence data for 289 taxa. The matrix was analyzed using RAxML. Bootstrap (BS) analyses yield 100\% BS support for all subtribes except Stanhopeinae (87\%). Generic relationships within subtribes are highly resolved and are generally congruent with those presented in previous studies and as summarized in Genera Orchidacearum. Relationships among subtribes are largely unresolved. The Szlachetko generic classification of Maxillariinae is not supported. A new combination is made for Maxillaria cacaoensis J.T.Atwood in Camaridium.
\end{abstract}

Key words: Orchidaceae, Cymbidieae, Maxillariinae, matK, ycfl, phylogenetics, Camaridium, Maxillaria cacaoensis, Vargasiella

Cymbidieae include many of the showiest Neotropical epiphytic orchids and an unparalleled diversity in floral rewards and pollination systems. Many researchers have posed questions such as "How many times and when has male euglossine bee pollination evolved?"(Ramírez et al. 2011), or "How many times have oil-reward flowers evolved?" (Reis et al. 2000) within this clade, but answering such questions requires a densely sampled and wellsupported phylogenetic hypothesis. Although the broad outlines of relationships within Cymbidieae were revealed by the $\mathrm{rbcL} / \mathrm{matK}$ analyses of Freudenstein et al. (2004) and summaries of Chase et al. (2003), both of these studies were constrained by low taxon sampling and low bootstrap support for many clades. The most recent publication of the Genera Orchidacearum series (Pridgeon 2009) provided a concise and authoritative summary of knowledge of this clade that includes 11 subtribes. Phylogenetic trees for Neotropical Cymbidieae published in that volume were based upon our nrITS/matK/ycfl data sets that were unpublished and included many sequences not deposited in GenBank. In attempting to rework these data for publication, we decided that attempting to align nrITS sequences across the entire tribe was unrealistic due to high levels of sequence divergence, and instead to concentrate our efforts on assembling a larger plastid data set based on two regions ( $m a t K$ and $y c f l$ ) that are among the most variable plastid exon regions and can be aligned with minimal ambiguity across broad taxonomic spans. Although various plastid spacer regions such as trnL-F or atpB$r b c L$ are more rapidly evolving (Shaw et al. 2005), they (like nrITS) are difficult or impossible to align with confidence across Cymbidieae. In this paper, we present phylogenetic analyses of ca. 280 taxa of Cymbidieae including representatives of 10 subtribes and most genera (excluding many Oncidiinae) utilizing the majority of the matK exon and a ca. 1500 base pair (bp) portion of the 3' end of $y c f 1$. Phylogenetic relationships within Oncidiinae were addressed in detail by Neubig et al. (2012). Relationships within Maxillariinae were studied using $\operatorname{nrITS/matK/atpB-}$ $r b c L$ spacer by Whitten et al.(2007), and the Bifrenaria clade was analyzed in more detail using nrITS/trnL-F by Koehler et al. (2002). Zygopetalinae relationships were previously studied using nrITS/matK/trnL-F (Neubig et al., 2009b; Whitten et al., 2005).

\footnotetext{
* This paper was prepared in the framework of the celebration of Lankester Botanical Garden's $40^{\text {th }}$ anniversary.
} 


\section{Methods}

Most matK sequences were downloaded from GenBankfrompreviousstudiesofCymbidieaesubtribes. Additional sequences were generated using primers matK-19F (CGTTCTGACCATATTGCACTATG) and $m a t K$ 1520R (CGGATAATGTCCAAATACCAAATA) and the amplification and sequencing protocols of Whitten et al. (2007). A ca. 1500 bp portion of 3' portion of $y c f l$ was amplified and sequenced using the primers and protocols in Neubig et al. (2009a). A list of taxa, vouchers, and GenBank numbers is presented in Table 1. Matrices were aligned using Muscle (Edgar, 2004)followed by manual adjustment of gaps to maintain reading frame using Se-Al (Rambaut 1996). The matK matrix was trimmed to eliminate a region of ambiguous alignment in the first $100 \mathrm{bp}$. Polystachya was chosen as the outgroup based upon broader sampling (Neubig et al. 2009a). Matrices and a list of vouchers are deposited in the Dryad Digital Repository (http://doi.org/10.5061/dryad.2rm60) or are available from the author. The resulting combined matrix consists of 288 ingroup taxa and 1 outgroup (Polystachya); the aligned matrix consists of 3618 characters (1605 for matK; 2013 for $y c f l)$. Gaps are coded as missing data.

The aligned matrix was analyzed using maximum likelihood (ML) as implemented in RAxML-HPC Blackbox version 7.6.3 (Stamatakis, 2006) via the CIPRES Science Gateway computing facility (http:// www.phylo.org/index.php/portal/). Analyses were run using default values with 200 fast bootstrap replicates. The resulting bootstrap trees were saved to a treefile, opened in PAUP* (Swofford 2003), and a majorityrule consensus tree was generated to display bootstrap support values. FigTree 1.4.0 (Rambaut 2013) was used to edit and print the best ML tree.

\section{Results and Discussion}

The resulting best ML tree is presented in Figures $1-5$; bootstrap (BS) values above $75 \%$ are annotated on this tree. Overall, the tree agrees well with previous studies based on plastid and nuclear regions (Górniak et al. 2010). Subtribe Cymbidiinae is represented by only a single taxon (Cymbidium); it is sister to all remaining taxa. All subtribes (as delimited in Genera Orchidacearum) received 100\% BS support (except for Stanhopeinae), but most relationships among subtribes lack BS support.

Eulophiinae - (Fig. 1). Out of the nine genera recognized in this subtribe, our sampling included the only two Neotropical genera; the majority of species are from the tropics of Africa, Madagascar, Asia, and Australia. Eulophiinae are weakly sister to Catasetinae in the single ML tree.

Catasetinae - (Fig. 1). Recent molecular phylogenetic studies (Batista et al., In press) place the three species of Cyanaeorchis Barb.Rodr. in Catasetinae; it is sister to Grobya Lindl., and they are sister to all remaining Catasetinae. Our sampling includes five of the seven genera, with Grobya and Mormodes absent; other phylogenetic studies confirm Grobya as monophyletic and a member of Catasetinae (Monteiro et al. 2010). Unpublished $y c f 1$ and $m a t K$ sequences for Cyanaeorchis arundinae (Rchb.f.) Barb.Rodr. and unidentified Grobya species (Whitten and Batista, unpubl.) confirm these relationships. Oscar Peréz (pers. comm.) also reported finding plastid/nuclear incongruence among sections of Cycnoches.

Cyrtopodiinae - (Fig. 1). Our analyses confirm the distinctiveness of this monogeneric subtribe from the vegetatively similar Catasetinae; Cyrtopodium is weakly sister to all remaining Cymbidieae, and not to Catasetinae, confirming the relationships found by Pridgeon and Chase (Pridgeon \& Chase 1998).

Oncidiinae - (Fig. 1). Our sampling of Oncidiinae was minimal, including placeholder representatives of the major clades within the subtribe; a much more extensive sampling based on matK/ycfl plus other regions was presented by Neubig et al. (2012). Oncidiinae are in a highly supported clade that includes subtribes Eriopsidinae, Zygopetalinae, Stanhopeinae, Coeliopsidinae, and Maxillariinae but relationships within this clade are poorly supported.

Eriopsidinae - (Fig. 2). Dressler (1981) included Eriopsis in Cyrtopodiinae on the basis of floral traits and pollinarium structure but later regarded it as incertae sedis (Dressler 1993). Szlachetko (1995) created a subtribe to accommodate this anomalous genus; our trees confirm its uniqueness relative to other subtribes. 
TABLE 1. List of taxa sequenced with GenBank numbers and voucher information.

\begin{tabular}{|c|c|c|c|}
\hline Taxon & matK & ycf1 & Voucher:Herbarium \\
\hline Acineta chrysantha (C. Morren) Lindl. & KF660253 & KF660372 & Whitten 91360 (FLAS) \\
\hline Acineta superba (Kunth) Rchb.f. & KF660254 & KF660523 & Whitten 3378 (FLAS) \\
\hline Aetheorhyncha andreettae (Jenny) Dressler & AY869932 & KF660386 & Dressler 6360 (FLAS) \\
\hline Aganisia fimbriata Rchb.f. & AY870006 & KF660404 & Breuer s.n. (M) \\
\hline Aganisia pulchella Lindl. & AY870007 & KF660403 & Breuer s.n. (M) \\
\hline Anguloa hohenlohii C. Morren & AF239429 & KF660512 & Whitten 3023 (FLAS) \\
\hline Anguloa uniflora Ruiz \& Pav. & KF660255 & KF660364 & Whitten 3263 (FLAS) \\
\hline Batemannia lepida Rchb.f. & AY869990 & KF660323 & Gerlach 92-3900 (M) \\
\hline Benzingia cornuta (Garay) Dressler & AY869927 & KF660450 & Whitten 1818 (FLAS) \\
\hline Benzingia estradae (Dodson) Dodson & AY869930 & KF660398 & Gerlach 96-4287 (M) \\
\hline Benzingia hajekii (D.E.Benn. \& Christenson) Dressler & AY869929 & KF660377 & Whitten 1751 (FLAS) \\
\hline Benzingia reichenbachiana (Schltr.) Dressler & AF239421 & KF660363 & Whitten 1747 (FLAS) \\
\hline Bifrenaria inodora Lindl. & DQ210744 & KF660365 & Whitten 0097 (FLAS) \\
\hline Bifrenaria tetragona (Lindl.) Schltr. & DQ210751 & KF660529 & Whitten 0506 (FLAS) \\
\hline Bifrenaria tyrianthina (Lodd. ex Loudon) Rchb.f. & DQ210752 & KF660379 & Whitten 0507 (FLAS) \\
\hline Braemia vittata (Lindl.) Jenny & AF239476 & KF660338 & Chase 84748 (FLAS) \\
\hline $\begin{array}{l}\text { Brasiliorchis gracilis (Lodd.) R. B. Singer, S. Koehler } \\
\text { \& Carnevali }\end{array}$ & DQ210811 & KF660426 & Whitten 2303 (FLAS) \\
\hline $\begin{array}{l}\text { Brasiliorchis schunkeana (Campacci \& Kautsky) } \\
\text { R. B. Singer, S. Koehler \& Carnevali }\end{array}$ & DQ210799 & KF660421 & Whitten 1992 (FLAS) \\
\hline Brassia aurantiaca (Lindl.) M.W.Chase & AF239492 & FJ563573 & Williams s.n. (FLAS) \\
\hline Brassia jipijapensis Dodson \& N.H.Williams & FJ564762 & FJ563258 & Whitten 1829 (FLAS) \\
\hline Camaridium bradeorum Schltr. & DQ210963 & KF660468 & Whitten 2639 (FLAS) \\
\hline Camaridium carinatum (Barb.Rodr.) Hoehne & DQ210828 & KF660431 & Whitten 2337 (FLAS) \\
\hline Camaridium ctenostachys (Rchb.f.) Schltr. & DQ210967 & KF660471 & Whitten 2647 (FLAS) \\
\hline Camaridium cucullatum (Lindl.) M.A Blanco & DQ210753 & KF660354 & Whitten 2547 (FLAS) \\
\hline Camaridium dendrobioides Schltr. & DQ210952 & KF660463 & Whitten 2627 (FLAS) \\
\hline Camaridium horichii (Senghas) M.A. Blanco & DQ210937 & KF660461 & Whitten 2602 (FLAS) \\
\hline Camaridium nutantiflorum Schltr. & DQ210964 & KF660469 & Whitten 2643 (FLAS) \\
\hline Camaridium ochroleucum Lindl. & DQ210626 & KF660312 & Gerlach 2003-3648 (M) \\
\hline Camaridium paleatum (Rchb.f.) M.A.Blanco & DQ210907 & KF660458 & Whitten 2561 (FLAS) \\
\hline Camaridium scalariforme (J.T.Atwood) M.A.Blanco & DQ210957 & KF660466 & Whitten 2633 (FLAS) \\
\hline Camaridium vestitum (Sw.) Lindl. & DQ209866 & KF660304 & Atwood \& Whitten 5070 (SEL) \\
\hline Catasetum expansum Rchb.f. & KF660256 & KF660525 & Whitten 3543 (FLAS) \\
\hline Chaubardia klugii (C.Schweinf.) Garay & AY869973 & KF660378 & Whitten 1853 (FLAS) \\
\hline Chaubardia surinamensis Rchb.f. & AY869974 & KF660309 & Gerlach 2001-2159 (M) \\
\hline Chaubardiella pubescens Ackerman & AY869944 & KF660416 & Whitten 1620 (FLAS) \\
\hline Chaubardiella subquadrata (Schltr.) Garay & AY869945 & KF660407 & Whitten s.n. (FLAS) \\
\hline Chaubardiella tigrina (Garay \& Dunst.) Garay & AY869946 & KF660311 & Gerlach 1651 (M) \\
\hline Chondrorhyncha hirtzii Dodson & AY869916 & KF660389 & Whitten 1637 (FLAS) \\
\hline Chondrorhyncha hirtzii Dodson & AY869913 & KF660406 & Maduro \& Olmos 217 (FLAS) \\
\hline Chondrorhyncha rosea Lindl. & AY869914 & KF660385 & Whitten 1760 (FLAS) \\
\hline
\end{tabular}


TABle 1. Continues.

\begin{tabular}{|c|c|c|c|}
\hline Taxon & matK & ycf1 & Voucher:Herbarium \\
\hline Chondroscaphe amabilis (Schltr.) Senghas \& G.Gerlach & AY869966 & KF660391 & Whitten 1855 (FLAS) \\
\hline Chondroscaphe bicolor (Rolfe) Dressler & AY869971 & KF660390 & Dressler ex Hoffman s.n. (FLAS) \\
\hline Chondroscaphe eburnea (Dressler) Dressler & AY869915 & KF660408 & Dressler 6361 (FLAS) \\
\hline $\begin{array}{l}\text { Chondroscaphe flaveola (Linden \& Rchb.f.) Senghas } \\
\text { \& G.Gerlach }\end{array}$ & AY869969 & KF660320 & Gerlach 93-3342 (M) \\
\hline $\begin{array}{l}\text { Christensonella ferdinandiana (Barb.Rodr.) Szlach., Mytnik, } \\
\text { Górniak \& Smiszek }\end{array}$ & DQ210670 & KF660353 & Koehler 109 (UEC) \\
\hline $\begin{array}{l}\text { Christensonella nardoides (Kraenzl.) Szlach., Mytnik, } \\
\text { Górniak \& Smiszek }\end{array}$ & DQ210890 & KF660452 & Whitten 2502 (FLAS) \\
\hline Christensonella pacholskii (Christenson) S.Koehler & DQ210851 & KF660437 & Whitten 2393 (FLAS) \\
\hline Cirrhaea fuscolutea Lindl. & KF660257 & KF660508 & Whitten 2976 (FLAS) \\
\hline Cirrhaea seidelii Pabst & KF660258 & KF660333 & Gerlach s.n. (M) \\
\hline Cischweinfia pusilla (C.Schweinf.) Dressler \& N.H.Williams & FJ565130 & FJ563799 & Whitten 3300 FLAS \\
\hline Clowesia dodsoniana E.Aguirre & KF660259 & KF660524 & Whitten 3542 (FLAS) \\
\hline Cochleanthes flabelliformis (Sw.) R.E.Schult. \& Garay & AY869965 & KF660513 & Whitten 99113 (FLAS) \\
\hline Coeliopsis hyacinthosma Rchb.f. & AF239440 & KF660337 & Whitten 93153 (FLAS) \\
\hline Comparettia falcata Poepp. \& Endl. & FJ563869 & FJ563283 & Williams N084 (FLAS) \\
\hline Comparettia macroplectron Rchb.f. \& Triana & FJ565135 & FJ563804 & Whitten 3425 (FLAS) \\
\hline Coryanthes elegantium Linden \& Rchb.f. & KF660260 & KF660318 & Whitten 87267 (FLAS) \\
\hline Coryanthes macrantha (Hook.) Hook. & KF660261 & KF660319 & Gerlach O-21458 (M) \\
\hline Coryanthes verrucolineata G.Gerlach & KF660262 & KF660317 & Gerlach 96-4284 (M) \\
\hline Cryptarrhena guatemalensis Schltr. & AY869983 & KF660476 & Pupulin \& Campos 2957 (USJ) \\
\hline Cryptarrhena lunata R.Br. & AY869982 & KF660405 & Whitten 98000 (FLAS) \\
\hline Cryptocentrum beckendorfii Carnevali & KF660263 & KF660307 & Beckendorf s.n. (CICY) \\
\hline Cryptocentrum peruvianum (Cogn.) C.Schweinf. & DQ210820 & KF660430 & Whitten 2322 (FLAS) \\
\hline Cryptocentrum roseans (Schltr.) A.D.Hawkes & DQ210903 & KF660457 & Whitten 2554 (FLAS) \\
\hline Cycnoches cooperi Rolfe & KF660264 & KF660526 & Whitten 3591 (FLAS) \\
\hline Cycnoches lehmannii Rchb.f. & KF660265 & KF660328 & Whitten 87011 (FLAS) \\
\hline Cycnoches manoelae P.Castro \& Campacci & KF660266 & KF660310 & Gerlach 05-1231 (M) \\
\hline Cycnoches pachydactylon Schltr. & KF660267 & KF660316 & Gerlach 00-3414 (M) \\
\hline Cymbidium devonianum Paxton & KF660268 & KF660325 & Chase $87030(\mathrm{~K})$ \\
\hline Cyrtidiorchis alata (Ruiz \& Pav.) Rauschert & DQ210627 & KF660321 & Gerlach 94-4005 (M) \\
\hline Cyrtidiorchis alata (Ruiz \& Pav.) Rauschert & DQ211044 & KF660505 & Whitten 2932 (FLAS) \\
\hline Cyrtochilum serratum (Lindl.) Kraenzl. & FJ563842 & FJ562462 & Chase $0-032(\mathrm{~K})$ \\
\hline Cyrtopodium andersonii (Lamb. ex Andrews) R.Br. & KF660269 & KF660329 & Kew no voucher \\
\hline Cyrtopodium flavum (Nees) Link \& Otto ex Rchb. & KF660270 & KF660522 & Whitten 3377 (FLAS) \\
\hline Cyrtopodium longibulbosum Dodson \& G.A.Romero & KF660271 & KF660453 & Whitten 2504 (QCA) \\
\hline Daiotyla albicans (Rolfe) Dressler & AY869917 & KF660396 & Whitten 1932 (FLAS) \\
\hline Dichaea eligulata Folsom & EU123625 & EU123747 & Pupulin 1094 (USJ-L) \\
\hline Dichaea fragrantissima Folsom & EU123628 & EU123750 & Pupulin 4601 (USJ-L) \\
\hline Dichaea panamensis Lindl. & EU123650 & EU123772 & Whitten 2556 (FLAS) \\
\hline Dichaea trulla Rchb.f. & EU123671 & EU123792 & Whitten 2475 (FLAS) \\
\hline
\end{tabular}


TABle 1. Continues.

\begin{tabular}{|c|c|c|c|}
\hline Taxon & matK & ycf1 & Voucher:Herbarium \\
\hline Dipteranthus grandiflorus (Lindl.) Pabst & AF350587 & FJ563191 & Chase $0-103(\mathrm{~K})$ \\
\hline Dressleria dilecta (Rchb.f.) Dodson & AF239507 & EU490731 & Whitten F1046 (SEL) \\
\hline Dressleria fragrans Dodson & KF660272 & KF660327 & Dodson 8855 (SEL) \\
\hline Dressleria helleri Dodson & KF660273 & KF660326 & Hills 87145 (FLAS) \\
\hline Embreea herrenhusana (Jenny) Jenny & KF660275 & KF660314 & Gerlach 04-2526 (M) \\
\hline Embreea rodigasiana (Claes ex Cogn.) Dodson & KF660276 & KF660313 & Gerlach 05-2172 (M) \\
\hline Eriopsis biloba Lindl. & DQ210866 & EU490743 & Whitten 3327 (FLAS) \\
\hline Eriopsis biloba Lindl. & DQ210866 & KF660441 & Whitten 2439 (FLAS) \\
\hline Eriopsis biloba Lindl. & DQ461806 & KF660515 & Whitten 3153 (QCA) \\
\hline Erycina pusilla (L.) N.H.Williams \& M.W.Chase & FJ565025. & FJ563690 & Whitten 1771 FLAS \\
\hline Eulophia guineensis Lindl. & AF239509 & EU490745 & Whitten 99029 (FLAS) \\
\hline Eulophia petersii (Rchb.f.) Rchb.f. & KF660274 & KF660332 & Chase $22361(\mathrm{~K})$ \\
\hline Fernandezia cuencae (Rchb.f.) M.W.Chase & FJ565079 & KF660454 & Whitten 2537 (FLAS) \\
\hline Fernandezia sanguinea (Lindl.) Garay \& Dunst. & FJ565009 & FJ563674 & Whitten 1700 FLAS \\
\hline Fernandezia tica Mora-Ret. \& García Castro & FJ564944 & FJ563591 & Dressler \& Atwood s.n. FLAS \\
\hline Galeandra devoniana M.R.Schomb. ex Lindl. & KF660278 & KF660330 & Pupulin 1133 (JBL) \\
\hline Galeottia burkei (Rchb.f.) Dressler \& Christenson & AY869987 & KF660400 & Maguire \& Politi 28175 (AMES) \\
\hline Galeottia ciliata (Morel) Dressler \& Christenson & AY869989 & KF660401 & Breuer s.n. (M) \\
\hline Galeottia colombiana (Garay) Dressler \& Christenson & AY869986 & KF660397 & Gerlach 93-3396 (M) \\
\hline Gongora amparoana Schltr. & AF239481 & KF660367 & Whitten 91036 (FLAS) \\
\hline Gongora armeniaca (Lindl.) Rchb.f. & AF239482 & KF660334 & Hills 86143 (FLAS) \\
\hline Gongora armeniaca (Lindl.) Rchb.f. & AF239482 & KF660374 & Whitten F1636 (FLAS) \\
\hline Gongora escobariana Whitten & KF660279 & KF660347 & Whitten 95023 (FLAS) \\
\hline Gongora hirtzii Dodson \& N.H.Williams & KF660280 & KF660349 & Whitten 93109 (FLAS) \\
\hline Gongora ilense Whitten \& Jenny & AF239480 & KF660509 & Whitten 2982 (FLAS) \\
\hline Gongora portentosa Linden \& Rchb.f. & AF239485 & KF660341 & Bennett 5258 (FLAS) \\
\hline Gongora portentosa Linden \& Rchb.f. & KF660281 & KF660350 & Bennett 5279 (FLAS) \\
\hline Gongora seideliana Rchb.f. & KF660282 & KF660348 & Whitten F-1635 (FLAS) \\
\hline Gongora sphaerica Jenny & KF660283 & KF660331 & Whitten 2003 (FLAS) \\
\hline Gongora tridentata Whitten & AF239483 & KF660373 & Whitten 1083 (FLAS) \\
\hline Grandiphyllum robustissimum (Rchb.f.) Docha Neto & FJ563959 & FJ563597 & Whitten 1777 FLAS \\
\hline $\begin{array}{l}\text { Guanchezia maguirei (C.Schweinf.) G.A.Romero } \\
\text { \& G.Carnevali }\end{array}$ & KF660284 & KF660410 & Maguire \& Politi 27931 (AMES) \\
\hline Heterotaxis crassifolia Lindl. & DQ210897 & KF660455 & Whitten 2544 (FLAS) \\
\hline Heterotaxis equitans (Schltr.) Ojeda \& Carnevali & DQ210877 & KF660448 & Whitten 2483 (FLAS) \\
\hline Heterotaxis maleolens (Schltr.) Ojeda \& Carnevali & DQ209972 & KF660486 & Whitten 2764 (FLAS) \\
\hline $\begin{array}{l}\text { Heterotaxis santanae (Carnevali \& I.Ramírez) Ojeda } \\
\quad \& \text { Carnevali }\end{array}$ & DQ209973 & KF660487 & Whitten 2765 (FLAS) \\
\hline Heterotaxis valenzuelana (A.Rich.) Ojeda \& Carnevali & DQ210950 & KF660510 & Whitten 2620 (FLAS) \\
\hline Heterotaxis violaceopunctata (Rchb.f.) F.Barros & DQ210807 & KF660424 & Whitten 2294 (FLAS) \\
\hline Hintonella mexicana Ames & FJ564940 & FJ562874 & Whitten W513 (FLAS) \\
\hline Horichia dressleri Jenny & AF239458 & KF660340 & Whitten 93151 (FLAS) \\
\hline
\end{tabular}


TABle 1. Continues.

\begin{tabular}{|c|c|c|c|}
\hline Taxon & matK & ycf1 & Voucher:Herbarium \\
\hline Houlletia brocklehurstiana Lindl. & KF660285 & KF660335 & Gerlach s.n. (M) \\
\hline Houlletia odoratissima Linden ex Lindl. \& Paxton & KF660286 & KF660315 & Gerlach 97-3285 (M) \\
\hline Houlletia sanderi Rolfe & AF239467 & KF660376 & Whitten 93079 (FLAS) \\
\hline Houlletia tigrina Linden ex Lindl. & AF239466 & KF660375 & Whitten 91354 (FLAS) \\
\hline Huntleya wallisii (Rchb.f.) Rolfe & EU123674 & EU123796 & Whitten 88026 (FLAS) \\
\hline Hylaeorchis petiolaris (Schltr.) Carnevali \& G.A.Romero & DQ211020 & KF660352 & Whitten 2874 (FLAS) \\
\hline Inti bicallosa (Rchb.f.) M.A.Blanco & DQ209946 & KF660417 & Whitten 1677 (FLAS) \\
\hline Inti bicallosa (Rchb.f.) M.A.Blanco & DQ210960 & KF660467 & Whitten 2636 (FLAS) \\
\hline Inti chartacifolia (Ames \& C.Schweinf.) M.A.Blanco & DQ209942 & EU490750 & Whitten 1597 (FLAS) \\
\hline Inti chartacifolia (Ames \& C.Schweinf.) M.A.Blanco & DQ211000 & KF660485 & Whitten 2752 (FLAS) \\
\hline Ixyophora viridisepala (Senghas) Dressler & AY869942 & KF660418 & Whitten 1749 (FLAS) \\
\hline Kefersteinia excentrica Dressler \& Mora-Ret. & AY869934 & KF660507 & Dressler 6236 (FLAS) \\
\hline Kefersteinia maculosa Dressler & AY869938 & KF660422 & Whitten 1997 (FLAS) \\
\hline Kefersteinia microcharis Schltr. & AY869937 & KF660308 & Pupulin 252 (USJ) \\
\hline Kefersteinia trullata Dressler & AY869936 & KF660423 & Whitten 1998 (FLAS) \\
\hline Kegeliella atropilosa L.O.Williams \& A.H.Heller & AF239459 & KF660342 & Whitten 93101 (FLAS) \\
\hline Kegeliella kupperi Mansf. & AF263666 & KF660339 & Whitten F167 (FLAS) \\
\hline Koellensteinia graminea (Lindl.) Rchb.f. & AY870003 & KF660429 & Chase $159(\mathrm{~K})$ \\
\hline Lacaena spectabilis (Klotzsch) Rchb.f. & KF660287 & KF660346 & Whitten F-184 (FLAS) \\
\hline Lockhartia amoena Endres \& Rchb.f. & FJ564686 & FJ563116 & Blanco 1803 (FLAS) \\
\hline Lueckelia breviloba (Summerh.) Jenny & KF660288 & KF660382 & Gerlach 96-6072 (M) \\
\hline Lueddemannia pescatorei (Lindl.) Linden \& Rchb.f. & AF239472 & KF660488 & Gerlach 2003-1482(M) \\
\hline Lycaste aromatica (Graham) Lindl. & AF263669 & KF660322 & Freudenstein s.n. \\
\hline Lycomormium fiskei H.R.Sweet & AF239441 & KF660528 & Whitten 91340 (FLAS) \\
\hline $\begin{array}{l}\text { Mapinguari auyantepuiensis (Foldats) Carnevali \& } \\
\text { R.B.Singer }\end{array}$ & DQ210830 & KF660432 & Whitten 2347 (FLAS) \\
\hline $\begin{array}{l}\text { Mapinguari longipetiolatus (Ames \& C.Schweinf.) Carnevali } \\
\text { \& R.B.Singer }\end{array}$ & DQ210747 & KF660305 & Atwood \& Whitten 5075 (SEL) \\
\hline Maxillaria acostae Schltr. & DQ210965 & KF660470 & Whitten 2644 (FLAS) \\
\hline Maxillaria angustissima Ames, F.T.Hubb. \& C.Schweinf. & DQ210993 & KF660479 & Whitten 2735 (FLAS) \\
\hline Maxillaria augustae-victoriae F.Lehm.\&Kraenzl. & DQ211026 & KF660500 & Whitten 2893 (FLAS) \\
\hline Maxillaria brachybulbon Schltr. & DQ210773 & KF660414 & Whitten 1583 (FLAS) \\
\hline Maxillaria buchtienii Schltr. & DQ211047 & KF660506 & Whitten 2940 (FLAS) \\
\hline Maxillaria cacaoensis J.T.Atwood & KC747493 & KC747494 & Whitten 3362 (FLAS) \\
\hline Maxillaria calantha Schltr. & DQ210900 & KF660456 & Whitten 2550 (FLAS) \\
\hline Maxillaria canarina D.E.Benn. \& Christenson & KF660289 & KF660518 & Whitten 3256 (FLAS) \\
\hline Maxillaria chionantha J.T.Atwood & DQ210969 & KF660472 & Whitten 2649 (FLAS) \\
\hline Maxillaria confusa Ames \& C.Schweinf. & DQ210994 & KF660480 & Whitten 2736 (FLAS) \\
\hline Maxillaria dalessandroi Dodson & DQ211024 & KF660499 & Whitten 2889 (FLAS) \\
\hline Maxillaria dillonii D.E.Benn. \& Christenson & KF660290 & KF660496 & Whitten 2878 (FLAS) \\
\hline Maxillaria ecuadorensis Schltr. & DQ210989 & KF660478 & Whitten 2724 (FLAS) \\
\hline Maxillaria elegantula Rolfe & DQ210921 & KF660460 & Whitten 2576 (FLAS) \\
\hline
\end{tabular}


TABle 1. Continues.

\begin{tabular}{|c|c|c|c|}
\hline Taxon & matK & ycf1 & Voucher:Herbarium \\
\hline Maxillaria exaltata (Kraenzl.) C.Schweinf. & DQ210818 & KF660428 & Whitten 2317 (FLAS) \\
\hline Maxillaria gentryi Dodson & DQ210975 & KF660475 & Whitten 2656 (FLAS) \\
\hline Maxillaria grayi Dodson & KF660291 & KF660497 & Whitten 2879 (FLAS) \\
\hline Maxillaria guadalupensis Cogn. & DQ210775 & KF660415 & Whitten 1593 (FLAS) \\
\hline Maxillaria guadalupensis Cogn. & KF660292 & KF660445 & Whitten 2468 (FLAS) \\
\hline Maxillaria hennisiana Schltr. & DQ210918 & KF660459 & Whitten 2572 (FLAS) \\
\hline Maxillaria litensis Dodson & KF660293 & KF660498 & Whitten 2888 (FLAS) \\
\hline Maxillaria longipes Lindl. & DQ210999 & KF660484 & Whitten 2751 (FLAS) \\
\hline Maxillaria longissima Lindl. & DQ210996 & KF660482 & Whitten 2745 (FLAS) \\
\hline Maxillaria lueri Dodson & DQ210954 & KF660464 & Whitten 2629 (FLAS) \\
\hline Maxillaria meridensis Lindl. & DQ210870 & KF660443 & Whitten 2451 (FLAS) \\
\hline Maxillaria ochroleuca Lodd. ex Lindl. & DQ210844 & KF660435 & Whitten 2378 (FLAS) \\
\hline Maxillaria platypetala Ruiz \& Pav. & DQ211033 & KF660502 & Whitten 2909 (FLAS) \\
\hline Maxillaria porrecta Lindl. & DQ210948 & KF660462 & Whitten 2617 (FLAS) \\
\hline Maxillaria pulla Linden \& Rchb.f. & DQ210872 & KF660444 & Whitten 2459 (FLAS) \\
\hline Maxillaria silvana Campacci & DQ210997 & KF660483 & Whitten 2747 (FLAS) \\
\hline Maxillaria sp. nov. & KF660294 & KF660520 & Whitten 3337 (FLAS) \\
\hline Maxillaria splendens Poepp. \& Endl. & FJ565112 & FJ563781 & Whitten 2949 FLAS \\
\hline Maxillaria triloris E.Morren & DQ209887 & KF660411 & Blanco 1640 (USJ) \\
\hline Maxillaria triloris E.Morren & DQ211038 & KF660503 & Whitten 2917 (FLAS) \\
\hline Maxillariella arbuscula (Lindl.) M.A.Blanco \& Carnevali & DQ211013 & KF660491 & Whitten 2810 (FLAS) \\
\hline Maxillariella elatior (Rchb.f.) M.A.Blanco \& Carnevali & DQ210797 & KF660420 & Whitten 1986 (FLAS) \\
\hline Maxillariella oreocharis (Schltr.) M.A.Blanco \& Carnevali & DQ210971 & KF660473 & Whitten 2652 (FLAS) \\
\hline Maxillariella ponerantha (Rchb.f.) M.A.Blanco \& Carnevali & DQ210973 & KF660474 & Whitten 2654 (FLAS) \\
\hline Maxillariella procurrens (Lindl.) M.A.Blanco \& Carnevali & DQ210854 & KF660438 & Whitten 2397 (FLAS) \\
\hline $\begin{array}{l}\text { Maxillariella variabilis (Bateman ex Lindl.) M.A.Blanco } \\
\text { \& Carnevali }\end{array}$ & DQ210995 & KF660481 & Whitten 2737 (FLAS) \\
\hline Miltonia regnellii Rchb.f. & AF239491 & FJ563571 & Chase $86059(\mathrm{~K})$ \\
\hline Mormolyca peruviana C.Schweinf. & DQ210885 & KF660451 & Whitten 2497 (FLAS) \\
\hline Mormolyca polyphylla Garay \& Wirth & DQ211009 & KF660489 & Whitten 2789 (FLAS) \\
\hline Mormolyca richii (Dodson) M.A.Blanco & DQ210836 & KF660434 & Whitten 2362 (FLAS) \\
\hline Mormolyca ringens (Lindl.) Gentil & DQ210680 & KF660493 & Whitten 2857 (FLAS) \\
\hline Mormolyca schlimii (Linden \& Rchb.f.) M.A.Blanco & DQ210847 & KF660436 & Whitten 2386 (FLAS) \\
\hline $\begin{array}{l}\text { Neogardneria murrayana (Gardner ex Hook.) Schltr. } \\
\text { ex Garay }\end{array}$ & AY869997 & KF660402 & Gerlach s.n. (M) \\
\hline Neomoorea wallisii (Rchb.f.) Schltr. & DQ210743 & EU490754 & Whitten 3010 (FLAS) \\
\hline Nitidobulbon nasutum (Rchb. f.) I.Ojeda \& Carnevali & DQ210756 & KF660419 & Whitten 1869 (FLAS) \\
\hline Nitidobulbon proboscideum (Rchb. f.) I.Ojeda \& Carnevali & DQ209857 & KF660303 & Atwood \& Whitten 5056 (SEL) \\
\hline Notyliopsis beatricis P.Ortiz & FJ565086 & FJ563753 & Whitten 2674 FLAS \\
\hline Oeceoclades maculata (Lindl.) Lindl. & KF660295 & KF660519 & Whitten 3333 (FLAS) \\
\hline Oncidium cirrhosum (Lindl.) Beer & FJ563845 & FJ562480 & Chase $86235(\mathrm{~K})$ \\
\hline Oncidium sphacelatum Lindl. & FJ563863 & FJ563267 & Whitten 3467 (FLAS \\
\hline
\end{tabular}


TABle 1. Continues.

\begin{tabular}{|c|c|c|c|}
\hline Taxon & matK & ycf1 & Voucher:Herbarium \\
\hline $\begin{array}{l}\text { Oncidium weinmannianum (Königer) M.W. Chase } \\
\text { \& N.H. Williams }\end{array}$ & FJ565036 & FJ563701 & Whitten 2328 FLAS \\
\hline Ornithidium aggregatum Rchb.f. & DQ210880 & KF660449 & Whitten 2488 (FLAS) \\
\hline Ornithidium canarense (J.T.Atwood) M.A.Blanco \& Ojeda & DQ209959 & KF660440 & Whitten 2437 (FLAS) \\
\hline Ornithidium coccineum (Jacq.) Salisb. ex R.Br. & DQ209875 & KF660494 & Whitten 2860 (FLAS) \\
\hline Ornithidium donaldeedodii Ackerman \& Whitten & KF660296 & KF660527 & Forbes s.n. (UC) \\
\hline Ornithidium fulgens Rchb.f. & DQ209968 & KF660465 & Whitten 2630 (FLAS) \\
\hline Ornithidium giganteum Lindl. & DQ210817 & KF660427 & Whitten 2316 (FLAS) \\
\hline Ornithidium multicaule (Poepp. \& Endl.) Rchb.f. & DQ211032 & KF660501 & Whitten 2905 (FLAS) \\
\hline Ornithidium serrulatum Lindl. & DQ211010 & KF660490 & Whitten 2800 (FLAS) \\
\hline Ornithidium sophronitis Rchb.f. & DQ210809 & KF660425 & Whitten 2296 (FLAS) \\
\hline Ornithocephalus dalstroemii (Dodson) Toscano \& Dressler & FJ564705 & FJ563134 & Blanco 2980 FLAS \\
\hline Ornithocephalus dalstroemii (Dodson) Toscano \& Dressler & FJ564705 & FJ563761 & Blanco 2980 FLAS \\
\hline Ornithocephalus inflexus Lindl. & DQ315891 & FJ563120 & Blanco 2545 (FLAS) \\
\hline Otoglossum globuliferum (Kunth) N.H.Williams \& M.W.Chase & FJ564700 & FJ563129 & Blanco 2856 (FLAS) \\
\hline Otoglossum globuliferum (Kunth) N.H.Williams \& M.W.Chase & FJ564700 & FJ563129 & Blanco 2856 FLAS \\
\hline Otostylis lepida (Linden \& Rchb.f.) Schltr. & AY870009 & KF660399 & Gerlach 94-968 (M) \\
\hline Otostylis paludosa (Cogn.) Schltr. & KF660297 & KF660517 & Whitten 3250 (FLAS) \\
\hline Paphinia clausula Dressler & KF660298 & EU490758 & Whitten 3600 (FLAS) \\
\hline Paphinia neudeckeri Jenny & AF239471 & KF660371 & Whitten 88041 (FLAS) \\
\hline Peristeria elata Hook. & AF239442 & EU490761 & Whitten 90158 (FLAS) \\
\hline Pescatoria cerina (Lindl. \& Paxton) Rchb.f. & AY869952 & KF660384 & Whitten s.n. (FLAS) \\
\hline Pescatoria coronaria Rchb.f. & AY869954 & KF660368 & Whitten 1758 (FLAS) \\
\hline Pescatoria lamellosa Rchb.f. & AY869953 & KF660369 & Whitten 1755 (FLAS) \\
\hline Pescatoria lawrenceana (Rchb.f.) Dressler & AF350662 & KF660393 & Whitten 1636 (FLAS) \\
\hline Pescatoria lehmannii Rchb.f. & AF239422 & KF660492 & Whitten 2848 (FLAS) \\
\hline Pescatoria pulvinaris (Rchb.f.) Dressler & AY869950 & KF660388 & Whitten 1748 (FLAS) \\
\hline Pityphyllum huancabambae (Kraenzl.) Whitten & DQ209957 & KF660439 & Whitten 2402 (FLAS) \\
\hline Pityphyllum laricinum (Kraenzl.) Schltr. & DQ209961 & KF660446 & Whitten 2473 (FLAS) \\
\hline Pityphyllum saragurense (Dodson) Whitten & DQ461805 & KF660514 & Whitten 3084 (QCA) \\
\hline Polycycnis gratiosa Endres \& Rchb.f. & AF239469 & EU490769 & Whitten 93178 (FLAS) \\
\hline Polycycnis gratiosa Endres \& Rchb.f. & AF239469 & EU490769 & Whitten 93178 (FLAS) \\
\hline Polyotidium huebneri (Mansf.) Garay & FJ563960 & FJ563598 & Romero 3124 AMES \\
\hline Polystachya cultriformis (Thouars) Lindl. ex Spreng. & DQ091312 & KF660306 & Carlsward 213 (SEL) \\
\hline Promenaea stapelioides (Link \& Otto) Lindl. & AY870002 & EU123797 & Whitten 94102 (FLAS) \\
\hline Promenaea xanthina (Lindl.) Lindl. & AY870000 & KF660366 & Whitten 1860 (FLAS) \\
\hline $\begin{array}{l}\text { Psychopsiella limminghei (E.Morren ex Lindl.) Lückel } \\
\text { \& Braem }\end{array}$ & FJ565152 & FJ563820 & Whitten 3561 FLAS \\
\hline Psychopsis sanderae (Rolfe) Lückel \& Braem & FJ564712 & FJ563158 & Chase $86126(\mathrm{~K})$ \\
\hline Rhetinantha acuminata (Lindl.) M.A.Blanco & DQ210981 & KF660477 & Whitten 2698 (FLAS) \\
\hline Rhetinantha notylioglossa (Rchb.f.) M.A.Blanco & DQ210645 & KF660351 & Koehler 0033 (UEC) \\
\hline
\end{tabular}


TABle 1. Continues.

\begin{tabular}{|c|c|c|c|}
\hline Taxon & matK & ycf1 & Voucher:Herbarium \\
\hline $\begin{array}{l}\text { Rossioglossum krameri (Rchb. f.) M.W. Chase } \\
\text { \& N.H. Williams }\end{array}$ & FJ563847 & FJ562488 & Chase $83166(\mathrm{~K})$ \\
\hline Rudolfiella floribunda (Schltr.) Hoehne & AF239433 & EU490776 & Whitten 97020 (FLAS) \\
\hline Rudolfiella sp. & FJ564977 & FJ563642 & Whitten 1618 FLAS \\
\hline Sauvetrea chicana (Dodson) M.A.Blanco & DQ461813 & KF660516 & Whitten 3187 (QCA) \\
\hline Sauvetrea chicana (Dodson) M.A.Blanco & KF660299 & KF660521 & Whitten 3338 (FLAS) \\
\hline Sauvetrea laevilabris (Lindl.) M.A.Blanco & DQ210832 & KF660433 & Whitten 2358 (FLAS) \\
\hline Schlimmia alpina Rchb.f. \& Warsz. & KF660300 & KF660345 & Bennett 5130 (MOL) \\
\hline Schlimmia stevensonii Dodson & AF239463 & KF660343 & Whitten 94107 (FLAS) \\
\hline Scuticaria hadwenii (Lindl.) Planch. & AF239424 & KF660370 & Whitten 97109 (FLAS) \\
\hline Scuticaria salesiana Dressler & DQ210875 & KF660447 & Whitten 2478 (FLAS) \\
\hline Sievekingia herrenhusana Jenny & AF239453 & KF660336 & Whitten 93010 (FLAS) \\
\hline Soterosanthus shepheardii (Rolfe) Jenny & AF239457 & EU490784. & Dodson 18580-3 (FLAS) \\
\hline Stanhopea anfracta Rolfe & AF239450 & KF660511 & Whitten 3022 (FLAS) \\
\hline Stanhopea annulata Mansf. & AF239444 & EU490786 & Whitten 87242 (FLAS) \\
\hline Stanhopea cirrhata Lindl. & AF239464 & KF660360 & Whitten F1296 (FLAS) \\
\hline Stanhopea confusa G.Gerlach \& Beeche & AF239449 & KF660359 & Whitten 94006 (FLAS) \\
\hline Stanhopea ecornuta Lem. & AF239445 & KF660362 & Whitten 90026 (FLAS) \\
\hline Stanhopea pulla Rchb.f. & AF239451 & KF660361 & Whitten 93117 (FLAS) \\
\hline Stanhopea tigrina Bateman ex Lindl. & FJ564736 & FJ563222 & Whitten 3585 FLAS \\
\hline Stenia bismarckii Dodson \& D.E.Benn. & AY869920 & KF660392 & Whitten 1698 (FLAS) \\
\hline Stenia calceolaris (Garay) Dodson \& D.E.Benn. & AY869919 & KF660394 & Whitten 1699 (FLAS) \\
\hline Stenotyla lankesteriana (Pupulin) Dressler & AY869962 & KF660383 & Dressler 6363 (FLAS) \\
\hline Stenotyla lendyana (Rchb.f.) Dressler & AY869963 & KF660381 & Dressler 6228 (FLAS) \\
\hline Stenotyla picta (Rchb.f.) Dressler & AY869961 & KF660395 & Dressler 6235 (FLAS) \\
\hline Sudamerlycaste ciliata (Ruiz \& Pav.) Archila & KF660301 & KF660495 & Whitten 2877 (FLAS) \\
\hline Telipogon hystrix (Dodson) N.H.Williams \& Dressler & DQ315899 & FJ563601 & Whitten 1824 (FLAS) \\
\hline Telipogon parvulus C.Schweinf. & DQ315909 & FJ563574 & Whitten 99259 (FLAS) \\
\hline Telipogon pogonostalix Rchb.f. & AF239488 & FJ562506 & Chase $0-123(\mathrm{~K})$ \\
\hline $\begin{array}{l}\text { Tolumnia gundlachii (C.Wright ex Griseb.) N.H.Williams } \\
\text { \& Ackerman }\end{array}$ & FJ565132 & FJ563801 & Whitten 3358 FLAS \\
\hline Tolumnia pulchella (Hook.) Raf. & FJ564820 & FJ563411 & Whitten 3499 (FLAS) \\
\hline Trevoria zahlbruckneriana (Schltr.) Garay & KF660302 & KF660324 & Dodson 17309 (MO) \\
\hline $\begin{array}{l}\text { Trichocentrum jonesianum (Rchb.f.) M.W.Chase } \\
\text { \& N.H.Williams }\end{array}$ & AF350653 & FJ562496 & Chase $86118(\mathrm{~K})$ \\
\hline Trichocentrum Iuridum (Lindl.) M.W.Chase \& N.H.Williams & FJ564957 & FJ563449 & Carnevali 6243 (CICY) \\
\hline Trichoceros antennifer (Humb. \& Bonpl.) Kunth & FJ564953 & FJ563612 & Whitten 1803 (FLAS) \\
\hline Trigonidium acuminatum Bateman ex Lindl. & DQ210640 & KF660358 & Koehler 363 (UEC) \\
\hline Trigonidium acuminatum Bateman ex Lindl. & DQ210867 & KF660442 & Whitten 2442 (FLAS) \\
\hline Trigonidium egertonianum Bateman ex Lindl. & DQ210714 & KF660356 & Koehler 317 (UEC) \\
\hline Trigonidium egertonianum Bateman ex Lindl. & DQ210730 & KF660357 & Koehler 361 (UEC) \\
\hline Trigonidium insigne Rchb.f. ex Benth. \& Hook.f. & DQ211041 & KF660504 & Whitten 2926 (FLAS) \\
\hline
\end{tabular}


TABLE 1. Continues.

\begin{tabular}{l|c|c|l}
\hline Taxon & matK & ycf1 & Voucher:Herbarium \\
\hline Trigonidium turbinatum Rchb.f. & DQ210713 & KF660355 & Koehler 315 (UEC) \\
\hline Trizeuxis falcata Lindl. & FJ563850 & FJ563198 & Chase O-129 (K) \\
\hline Vasqueziella boliviana Dodson & AF239473 & KF660344 & Vasquez s.n. (FLAS) \\
\hline Warczewiczella discolor (Lindl.) Rchb.f. & AY869959 & KF660412 & Whitten 1859 (FLAS) \\
\hline Warczewiczella marginata Rchb.f. & AY869958 & EU490794 & Whitten s.n. (FLAS) \\
\hline Warczewiczella wailesiana (Lindl.) E.Morren & AY869960 & KF660387 & Gerlach 93-3314 (M) \\
\hline Warrea warreana (Lodd. ex Lindl.) C.Schweinf. & AF239417 & EU123798 & Whitten 1752 (FLAS) \\
\hline Xylobium leontoglossum (Rchb.f.) Benth. ex Rolfe & DQ209970 & KF660413 & Whitten 2683 (FLAS) \\
\hline Xylobium pallidiflorum (Hook.) G.Nicholson & AF239434 & EU490795 & Whitten 1876 (FLAS) \\
\hline Xylobium zarumense Dodson & AF239435 & KF660380 & Whitten 1881 (FLAS) \\
\hline Zygopetalum maxillare Lodd. & AY869996 & FJ562864 & Whitten 94103 (FLAS) \\
\hline Zygosepalum tatei (Ames \& C.Schweinf.) Garay \& Dunst. & AY869994 & KF660409 & Maguire \& Politi 27494 (AMES) \\
\hline
\end{tabular}

Zygopetalinae - (Fig. 2). Our results are largely congruent with our previous study (Whitten et al. 2005) based on matK/trnL-F/ITS data, although there is less support for many genera. The nonmonophyly of Warczewiczella is unusual, and might be due to mislabeled DNA samples; resampling with new collections is needed. Relationships within Dichaea were clarified by Neubig et al. (2009b). Subtribe Vargasiellinae consists of one genus with two poorly collected species, one from the tepuis of Venezuela and the other from eastern Peru. We were unable to obtain DNA of these taxa. Dressler (1993) included Vargasiella C.Schweinf. in Zygopetalinae but suggested it might warrant subtribal status. RomeroGonzález and Carnevali (1993) validated the subtribal name and suggested that it should remain in its own subtribe pending better specimens and molecular data. Recent collections and DNA sequences of Vargasiella peruviana C.Schweinf. place it with high support in Zygopetalinae in an unresolved clade with Warrea warreana (Lodd. ex Lindl.) C.Schweinf. and Warreopsis spp. (Szlachetko et al., in press; M. Kolanowska, pers. comm.). Vargasiella is sister to Warrea but with weak support. These data confirm Dressler's intuition (Dressler 1993) regarding its subtribal position; therefore, Vargasiellinae should not be recognized.

Coeliopsidinae - (Fig. 3). Our sampling included one species of each of the three genera comprising this small subtribe. The subtribe is highly supported but weakly sister to Stanhopeinae in agreement with Whitten et al. (2000).

Stanhopeinae - (Fig. 3). Although the circumscription of generic boundaries within this subtribe are highly congruent with morphology-based classifications, this subtribe has the lowest BS support (87\%). The odd monotypic Braemia vittata (Lindl.) Jenny is sister to all other genera. These data are highly congruent with the trees of Whitten et al. (2000), but the placement of Sievekingia requires more study. In the Whitten et al. (2000) analyses based upon matK/trnL-F/ nrITS, Sievekingia is strongly sister to Coryanthes. In the plastid matK/ycfl trees, the single sample of Sievekingia is sister to the multiflowered clade of Stanhopea (creating a paraphyletic Stanhopea). More extensive sampling with nuclear and plastid regions is needed to resolve this, because it appears to be one of the few instances of conflict between nrITS and plastid trees in Cymbidieae. One possible source of error within the Stanhopea/Coryanthes/Sievekingia clade is from missing data in $y c f 1$ for Coryanthes; the $3720 \mathrm{~F}$ primer did not amplify for Coryanthes; consequently, about half of the $y c f 1$ sequence data are missing for Coryanthes species.

Maxillariinae - (Figs. 4, 5). Relationships within Maxillariinae were addressed in greater detail by Whitten et al. (2007), Blanco et al. (2007; 2008), and Blanco (2013) based upon a larger taxon sampling of $m a t K / n r I T S 1 \& 2 / a t p B-r b c L$ spacer. Our sampling with $m a t K / y c f 1$ was smaller (119 taxa vs. over 600), 


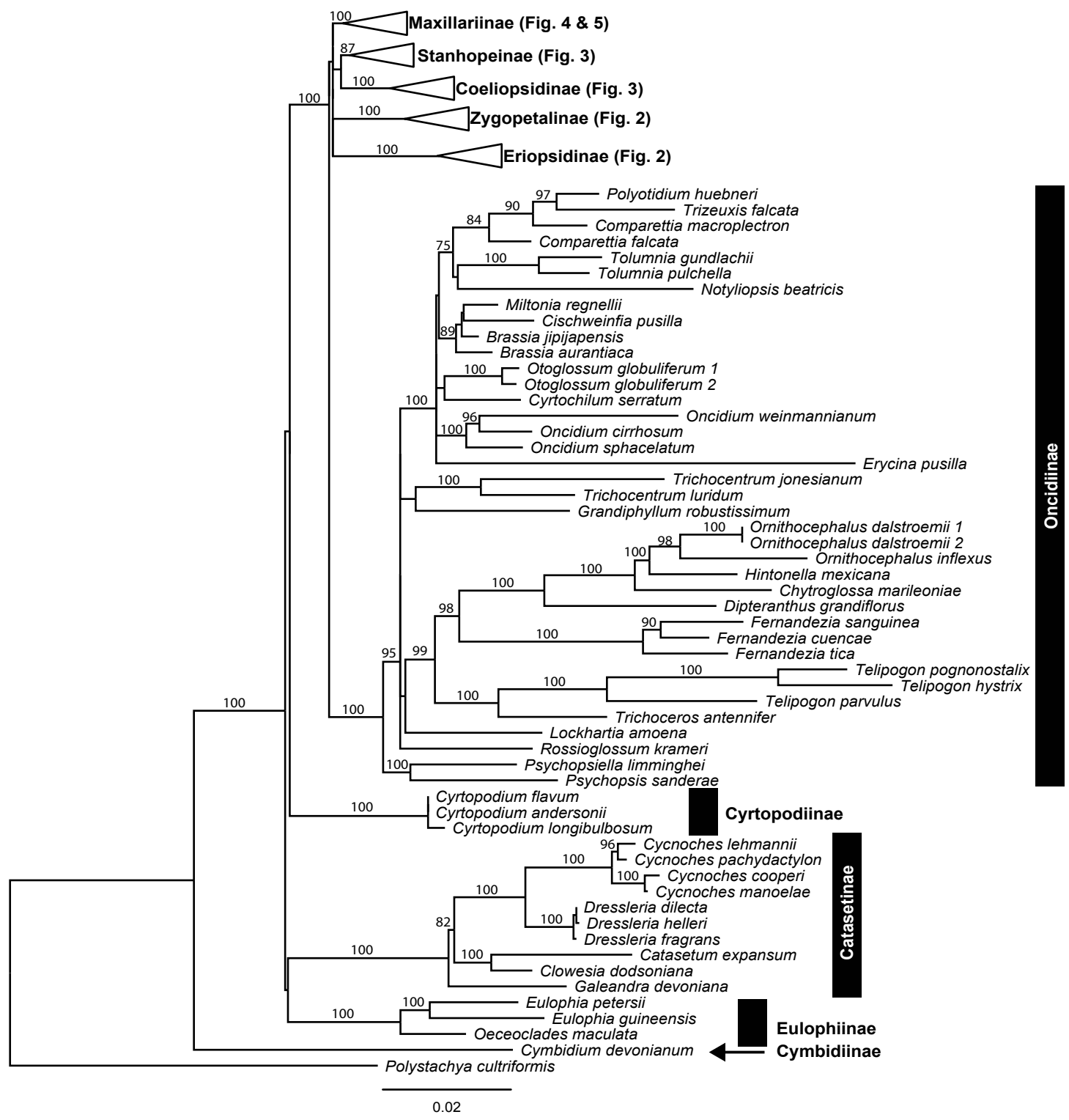

FIgURE 1. Best ML tree with bootstrap values added, showing Cymbidiinae, Eulophiinae, Catasetinae, Cyrtopodiinae, and Oncidiinae.

but the two data sets recovered the same major clades, supporting the generic concepts presented by Blanco et al. (2007). Our sampling included two individuals of several species; in each pair, there are nucleotide differences separating the two, indicating that $y c f 1 /$ $m a t K$ is often capable of resolving not only closely related species but also intraspecific variation.

Several taxa not present in the 2007 sampling were added to this study. These include Cryptocentrum beckendorfii Carnevali and Maxillaria cacaoensis J.T.Atwood. Cryptocentrum beckendorfii, an anomalous species with large pseudobulbs, is placed within Cryptocentrum with 100\% BS support (Fig. 5). Maxillaria cacaoensis was hypothesized by Atwood to be a member of the Camaridium cucullatum (Lindl.) M.A.Blanco clade (=Psittacoglossum La Llave \& Lex., but DNA samples of this rare taxon became available only recently. Maxillaria 


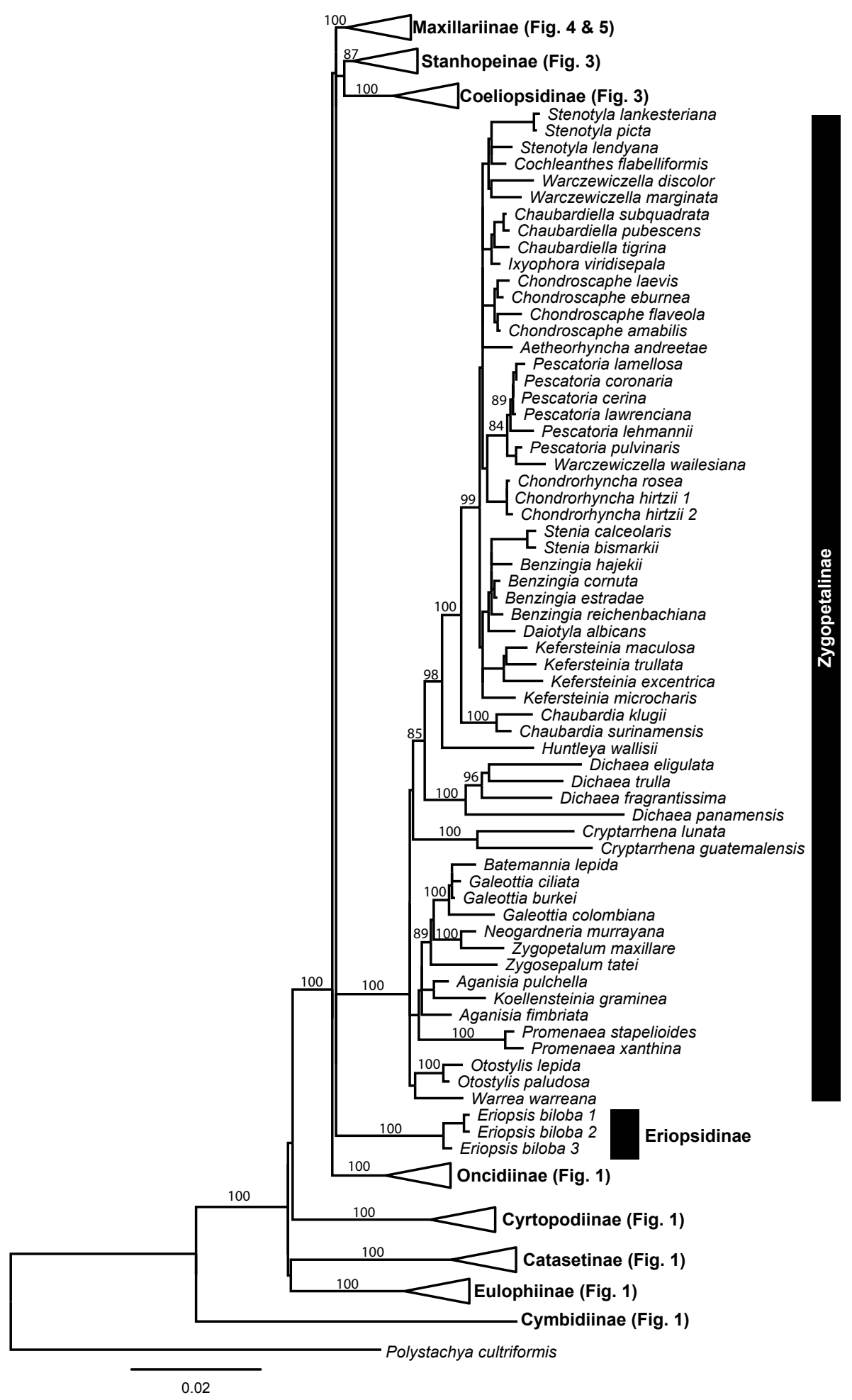

FIGURE 2. Best ML tree with bootstrap values added, showing Eriopsidinae and Zygopetalinae. 


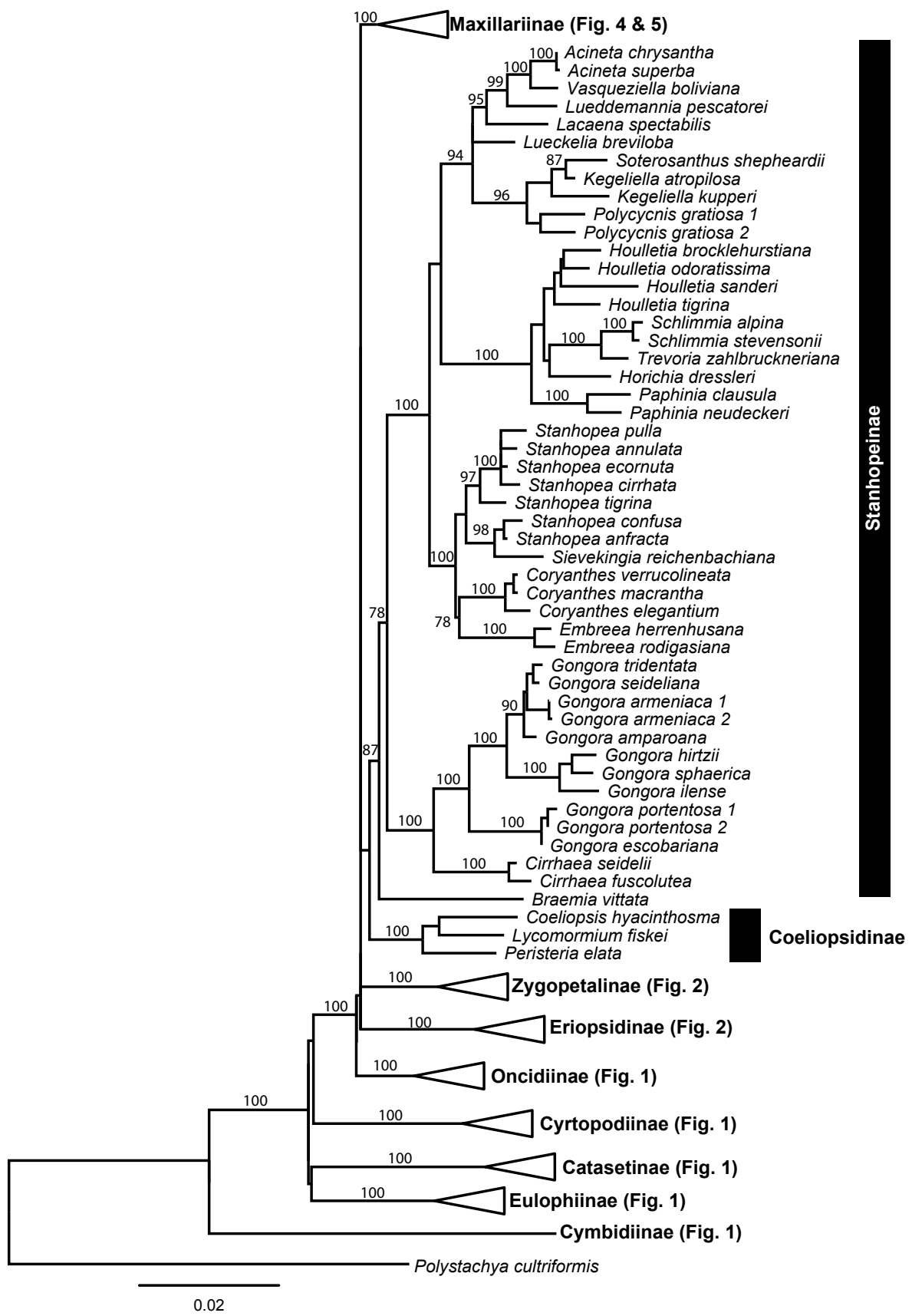

FIgURE 3. Best ML tree with bootstrap values added, showing Coeliopsidinae and Stanhopeinae.

cacaoensis is sister to C. cucullatum in our ycfl/ matK trees (Fig. 5), and requires a new combination in Camaridium. It was erroneously transferred to Mapinguari Carnevali \& R.B.Singer by Szlachetko et al. (2012). Morphologically, it resembles a dwarf
C. cucullatum, and the capsule has apical dehiscence, a trait shared by all Camaridium species for which we have observed mature fruits. Based upon these molecular and morphological data, we transfer this species to Camaridium. 


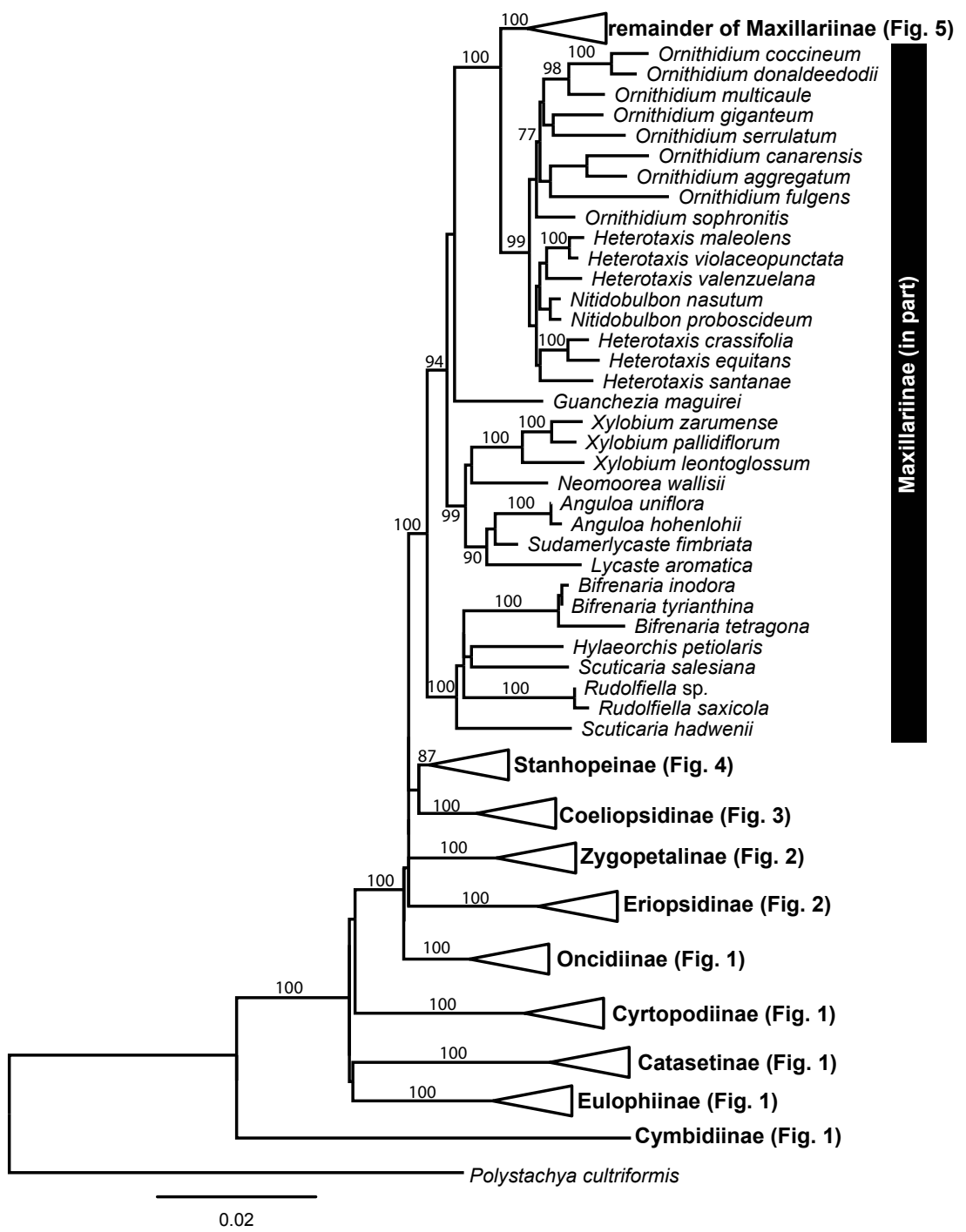

FiguRE 4. Best ML tree with bootstrap values added, showing basal portion of Maxillariinae.

Camaridium cacaoense (J.T.Atwood) Whitten, comb. nov.

Basionym: Maxillaria cacaoensis J.T.Atwood, Selbyana 19(2):254. 1999 (1998, pub. 1999). Mapinguari cacaoense (J.T.Atwood) Szlach. \& Sitko, Biodiv. Res. Conservation 25:30. 2012, syn. nov.

The Whitten et al. (2007) and Blanco et al. (2007) classifications of Maxillariinae were based upon analyses of $\operatorname{nrITS} / m a t K+\operatorname{trnK} / a t p B-r b c L$ spacer for over 600 individuals. An alternative classification was published by Szlachetko et al. (2012). The Szlachetko classification was based on analyses of a 249 -taxon nrITS matrix that is largely congruent (though less resolved) than the Whitten et al. trees. The resulting Szlachetko classification accepts most of the genera proposed by Blanco et al. (2007) but splits many of them to increase the number of genera from 17 to 37 .

A detailed, genus-by-genus critique of the Szlachetko et al. (2012) classification falls outside the scope of this paper, but we reject the generic concepts presented in their paper. Szlachetko and coworkers reject monophyly as a criterion for generic rank; therefore, many of their genera are paraphyletic 


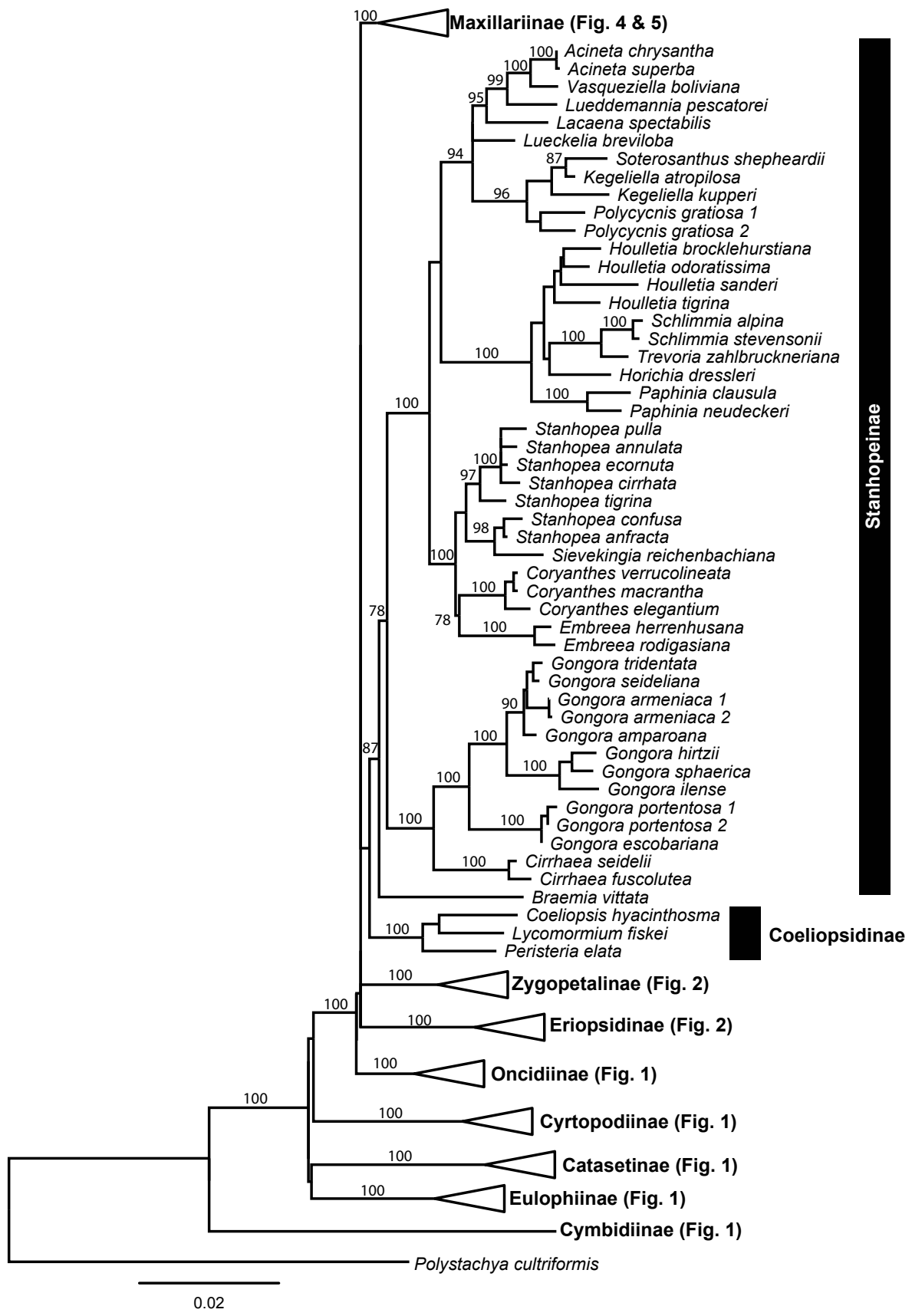

FIgURE 5. Best ML tree with bootstrap values added, showing remainder of Maxillariinae.

or polyphyletic as plotted onto any molecular or morphological tree and are based on idiosyncratically selected morphological characters (floral and/or vegetative), often without molecular data or with contradicting molecular evidence.
Many of their new genera consist of one or two morphologically odd species embedded within larger genera [e.g., Marsupiaria Hoehne = Heterotaxis valenzuelana (A.Rich.) Ojeda \& Carnevali; Vazquezella Szlach. \& Sitko = Heterotaxis 
equitans (Schltr.) Ojeda \& Carnevali; Chrysocycnis Linden \& Rchb.f., Anthosiphon Schltr., Hoehnella Szlach. \& Sitko $=$ Rhetinantha witseniodes (Schltr.) M.A.Blanco; Pseudocymbidium Szlach. \& Sitko = Maxillaria lueri Dodson]. Other genera are composed of 15 or more species that are monophyletic but are embedded within other genera (e.g. Calawaya Szlach. \& Sitko, embedded in Maxillaria Ruiz \& Pav.). Camaridium Lindl. is split into at least eight genera. Some segregates (e.g., Chaseopsis Szlach. \& Sitko) are based solely upon morphological traits and include species that were not included in either molecular study [e.g., Camaridium burgeri (J.T.Atwood) M.A.Blanco]. Their circumscription of Chaseopsis omits taxa that are sister to the generitype in the molecular trees but lack the defining essential "generic" characters (e.g., Maxillaria flava Ames, F.T.Hubb. \& C.Schweinf. = Camaridium ramonense (Schltr.) M.A.Blanco; Maxillaria lankesteri Ames = Camaridium aurantiacum (Schltr.) M.A.Blanco). The Szlachetko et al. classification produces genera that are easily suited to production of dichotomous keys, because any morphologically anomalous species are automatically placed into another genus. Because there is no objective basis for selecting "critical" characters that define genera, their classification is without merit.

\section{Conclusions}

The $m a t K / y c f 1$ data produce trees that are highly congruent with the classification presented in volume 5 Genera Orchidacearum. Most subtribes have high bootstrap support, and generic relationships are congruent with previous molecular studies. In comparison to plastid intron/spacer regions (e.g., $\operatorname{trn} L-F$, atpB-rbcL), these coding regions can be aligned with much more confidence across larger taxonomic groups (e.g., tribes), especially if they are aligned using amino acid translations. This combination also appears capable of providing species-level discrimination in some genera, although more detailed sampling is needed to evaluate this fully. In terms of sequencing ease and cost effectiveness vs. phylogenetic resolution, the combination of matK/ycfl/nrITS may prove efficient within Orchidaceae. Nevertheless, these plastid trees fail to provide resolution and support of relationships among subtribes.

Givnish et al. (2013) recently utilized complete plastomes to estimate phylogenetic relationships among 39 orchid taxa. Although only a few subtribes of Cymbidieae were represented in their data set, subtribal relationships were still unresolved. Their results imply that the addition of more plastid genes with the objective of resolving these nodes may be futile and that these relationships will only be resolved by the addition of nuclear data sets. Clearly, much more data are needed before we fully understand the patterns of evolution within Cymbidieae.

We hope to add more representatives of Cymbidiinae, Eulophiinae, and Catasetinae. Catasetinae might provide an excellent system for study of the evolution of diverse floral reward systems; it includes five genera that are all androeuglossophilous fragrance-reward flowers (Catasetum, Cycnoches, Clowesia, Dressleria, Mormodes); these five genera are sister to Galeandra, with nectar deceit flowers, to Grobya, with oil-reward flowers (Pansarin et al., 2009), and to Cyanaeorchis, with unknown pollinators.

Previous attempts to utilize molecular clock methods to estimate the age of subtribes within Cymbidieae (Ramírez et al. 2011) utilized more limited taxon sampling that was biased towards androeuglossophilous taxa. Our more complete sampling of generic relationships based on more sequence data might warrant reexamination of these age estimates.

AcKNOWLedGMENTs. We thank Günter Gerlach (Munich Botanical Garden), Ron Determann, Becky Brinkman (Atlanta Botanical Garden), Gustavo Romero (AMES), Andy and Harry Phillips (Andy's Orchids) and the Portilla family (Ecuagenera) for generous access and help in obtaining specimens and vouchers. Lorena Endara provided valuable assistance in Ecuador, and Mario Blanco and Robert Dressler (Lankester Garden) provided specimens and valuable discussions. Kent Perkins was invaluable in accessioning and imaging specimens. Marta Kolanowska generously shared unpublished information on the placement of Vargasiella. Alec Pridgeon provided valuable criticisms and edits. We thank Savita Shanker and Patrick Thimote at the Interdisciplinary Center for Biotechnology Research at UF for DNA sequencing services. 


\section{LITERATURE CITED}

Batista,J.A.N.,A.C.M.Mota,K.Proite,L.deBemBianchetti, G. A. Romero-Gonzalez, H. Huerta, and G. Salazar. In press. Molecular phylogenetics of Cyanaeorchis (Cymbidieae, Epidendroideae,Orchidaceae) and a new species from the cerrado of central and southeastern Brazil. Phytotaxa.

Blanco, M., G. Carnevali, W. M. Whitten, R. B. Singer, S. Koehler, N. H. Williams, I. Ojeda, K. Neubig, and L. Endara. 2007. Generic realignments in Maxillariinae (Orchidaceae). Lankesteriana 7:515-537.

Blanco, M. A. 2013. New combinations and synonyms in the Maxillariiinae (Orchidaceae). Selbyana 31:52-59.

Blanco, M. A., G. Carnevali, W. M. Whitten, R. B. Singer, S. Koehler, N. H. Williams, I. Ojeda, K. M. Neubig, and L. Endara. 2008. Generic realignments in Maxillariinae (Orchidaceae): Corrigenda et addenda. Lankesteriana $8: 15$.

Chase, M. W., K. M. Cameron, R. L. Barrett, and J. V. Freudenstein. 2003. DNA data and Orchidaceae systematics: a new phylogenetic classification. Pages 69-89 in Orchid Conservation (K. W. Dixon, S. P. Kell, R. L. Barrett, and P. J. Cribb, eds.). Natural History Publications, Kota Kinabalu, Sabah, Malaysia.

Dressler, R. L. 1981. The Orchids: Natural History and Classification. Harvard University Press, Cambridge, Massachusetts, USA.

Dressler, R. L. 1993. Phylogeny and Classification of the Orchid Family. Dioscorides Press, Portland, Oregon, USA.

Edgar, R. 2004. MUSCLE: a multiple sequence alignment method with reduced time and space complexity. doi:10.1186/1471-2105-5-113. BMC Bioinformatics 5: 113.

Freudenstein, J. V., C. van den Berg, D. H. Goldman, P. J. Kores, M. Molvray, and M. W. Chase. 2004. An expanded plastid DNA phylogeny of Orchidaceae and analysis of jackknife branch support strategy. American Journal of Botany 91:149-157.

Givnish, T. J., M. Ames, S. P. Lyon, K. M. Cameron, K. M. Neubig, W. M. Whitten, M. K. Arroyo, J. LeebensMack, M. A. Clemets, and N. H. Williams. Year. The 39 Steps: A plastome phylogeny for orchid tribes, with implications for the evolution of epiphytism, CAM photosynthesis, and net rates of species diversification in Monocots V: 5th International Conference on Comparative Biology of Monocotyledons, New York Botanical Gardens:48-49.

Górniak, M., O. Paun, and M. W. Chase. 2010. Phylogenetic relationships within Orchidaceae based on a low-copy nuclear coding gene, $X d h$ : Congruence with organellar and nuclear ribosomal DNA results. Molecular
Phylogenetics and Evolution 56:784-795.

Koehler, S., N. H. Williams, W. M. Whitten, and M. D. E. do Amaral. 2002. Phylogeny of the Bifrenaria (Orchidaceae) complex based on morphology and sequence data from nuclear rDNA internal transcribed spacers (ITS) and chloroplast trnL-trnF region. International Journal of Plant Sciences 163:1055-1066.

Monteiro, S. H. N., A. Selbach-Schnadelbach, R. P. d. Oliveira, and C. van den Berg. 2010. Molecular phylogenetics of Galeandra (Orchidaceae: Catasetinae) based on plastid and nuclear DNA Sequences. Systematic Botany 35:476-486.

Neubig, K. M., W. M. Whitten, B. S. Carlsward, M. A. Blanco, L. Endara, N. H. Williams, and M. Moore. 2009a. Phylogenetic utility of $y c f 1$ in orchids: a plastid gene more variable than matK. Plant Systematics and Evolution 277:75-84.

Neubig, K. M., W. M. Whitten, N. H. Williams, M. A. Blanco, L. Endara, J. G. Burleigh, K. Silvera, J. C. Cushman, and M. W. Chase. 2012. Generic recircumscriptions of Oncidiinae (Orchidaceae: Cymbidieae) based on maximum likelihood analysis of combined DNA datasets. Botanical Journal of the Linnean Society 168:117-146.

Neubig, K. M., N. H. Williams, W. M. Whitten, and F. Pupulin. 2009b. Molecular phylogenetics and the evolution of fruit and leaf morphology of Dichaea (Orchidaceae: Zygopetalinae). Annals of Botany 104:457-467.

Pansarin, L. M., M. D. Castro, and M. Sazima. 2009. Osmophore and elaiophores of Grobya amherstiae (Catasetinae, Orchidaceae) and their relation to pollination. Botanical Journal of the Linnean Society 159:408-415.

Pridgeon, A. M., and M. W. Chase. 1998. Phylogenetics of subtribe Catasetinae (Orchidaceae) from nuclear and chloroplast DNA sequences. Pages pp. 275-281 in Proceedings of the 15th World Orchid Conference (C. E. de Britto Pereira, ed.) Naturalia Publications, Turriers, France.

Pridgeon, A. M., P.J. Cribb, M. W. Chase, and F.N. Rasmussen. 2009. Genera Orchidacearum Volume 5 Epidendroideae (Part Two). Oxford University Press, Inc., New York.

Rambaut, A. 1996. Se-Al: Sequence Alignment Editor. Available at http://evolve.zoo.ox.ac.uk/.

Rambaut, A. 2013. FigTree, version 1.4.0.

Ramírez, S. R., T. Eltz, M. K. Fujiwara, G. Gerlach, B. Goldman-Huertas, N. D. Tsutsui, and N. E. Pierce. 2011. Asynchronous diversification in a specialized plant-pollinator mutualism. Science 333:1742-1746. 
Reis, M. G., A. D. de Faria, V. Bittrich, M. D. E. Amaral, and A. J. Marsaioli. 2000. The chemistry of flower rewards - Oncidium (Orchidaceae). Journal Of The Brazilian Chemical Society 11:600-608.

Romero-Gonzalez, G. A., and G. Carnevali. 1993. Reappraisal of subtribe Vargasiellinae (Maxillarieae, Orchidaceae). Novon 3:79-80.

Shaw, J., E. B. Lickey, J. T. Beck, S. B. Farmer, W. Liu, J. Miller, K. C. Siripun, C. T. Winder, E. E. Schilling, and R. L. Small. 2005. The tortoise and the hare II: relative utility of 21 noncoding chloroplast DNA sequences for phylogenetic analysis. Am. J. Bot. 92:142-166.

Stamatakis, A. 2006. RAxML-VI-HPC: maximum likelihood-based phylogenetic analyses with thousands of taxa and mixed models. Bioinformatics 22:26882690 .

Swofford, D. L. 2003. PAUP*: Phylogenetic Analysis Using Parsimony (*and other methods), version 4.0b10. Sinauer.

Szlachetko, D., M. Sitko, PTukallo, and J. MytnikEjsmont. 2012. Taxonomy of the subtribe Maxillariinae (Orchidaceae, Vandoideae) revised. Biodiversity Research and Conservation 25:13-38.

Szlachetko, D. L. 1995. Systema Orchidalium, Fragmenta
Floristica et Geobotanica. Supplementum 3. W. Szafer Institute of Botany, Polish Academy of Sciences, Kraków, Poland.

Szlachetko, D. L., M. Górniak, M. Kolanowska, J. MytnikEjsmont, A. K. Kowalkowska, and T. Koliński. in press. Taxonomic position of the genus Vargasiella (Orchidaceae, Vandoideae) based on molecular and morphological evidence. Molecular Phylogenetics and Evolution.

Whitten, M. W., N. H. Williams, R. L. Dressler, G. Gerlach, and F. Pupulin. 2005. Generic relationships of Zygopetalinae (Orchidaceae: Cymbideae): Combined molecular evidence. Lankesteriana 5:87-107.

Whitten, W. M., M. A. Blanco, N. H. Williams, S. Koehler, G. Carnevali, R. B. Singer, L. Endara, and K. M. Neubig. 2007. Molecular phylogenetics of Maxillaria and related genera (Orchidaceae: Cymbidieae) based on combined molecular data sets. American Journal of Botany 94:1860-1889.

Whitten, W. M., N. H. Williams, and M. W. Chase. 2000. Subtribal and generic relationship of Maxillarieae (Orchidaceae) with emphasis on Stanhopeinae: combined molecular evidence. American Journal of Botany 87:1842-1856. 